\title{
Real Belyi Theory
}

\author{
by \\ Bernhard KöcK and David Singerman
}

\section{Introduction}

Belyi's Theorem [Be] of 1979 had a profound effect on Galois Theory, Riemann surfaces and complex algebraic curves. It led Grothendieck $[\mathrm{Gr}]$ to develop his theory of dessins d'enfants in which there has been a great interest. The theory is about embedding graphs into compact Riemann surfaces. For further applications to topics such as such as moduli spaces and Physics the reader is recommended to consult [LZ]. The combinatorial and topological foundations of the theory, as well as the connection with Riemann surfaces, were developed at the same time by G. A. Jones and D. Singerman in [JS1].

The main purpose of this paper is to develop a Belyi type theory that applies to Klein surfaces, i.e. (possibly non-orientable) surfaces with boundary which carry a dianalytic structure. More precisely, let $S$ be a connected compact Klein surface. We prove that the following statements are equivalent with the possible exception that statement (i) may not be implied by the other statements if $S$ both has empty boundary and is non-orientable:

(i) $S$ can be defined over $\overline{\mathbb{Q}} \cap \mathbb{R}$.

(ii) $S$ admits a Belyi map $\beta: S \rightarrow \Delta$ where $\Delta$ denotes the compactifed closed upper half plane.

(iii) $S$ is isomorphic to the compactification $\overline{\mathcal{U} / L}$ of the quotient surface $\mathcal{U} / L$ for some subgroup $L$ of finite index in the extended modular group $\Gamma^{*}$ acting on the upper half plane $\mathcal{U}$. 
(iv) $S$ is isomorphic to the compactification $\overline{\mathcal{U} / M}$ for some subgroup $M$ of finite index in $\Gamma^{*}(2)$, the extended principal congruence subgroup of level 2.

(v) $S$ is isomorphic to the quotient surface $\mathcal{X} / K$ for some subgroup $K$ of finite index in an extended triangular group acting on one of the three simply connected Riemann surfaces $\mathcal{X}=\hat{\mathbb{C}}, \mathbb{C}$ or $\mathcal{U}$.

(vi) $S$ carries a map $\mathcal{M}$ such that $S$ is isomorphic to the quotient surface $\mathcal{X} / K$ where $K$ is the map subgroup for $\mathcal{M}$.

We refer the reader to the main body of this paper for a detailed definition of the various notions which have been used in the formulation of these statements. The equivalence between (i) and (ii), i.e. the real analogue of Belyi's theorem, is discussed in Section 2. In Section 3 we represent Klein surfaces as quotient surfaces. And finally, in Section 4, we develop a theory of maps on Klein surfaces; the combinatorial and topological foundations have been laid out by R. P. Bryant and D. Singerman in $[\mathrm{BS}]$.

Acknowledgements. We would like to thank Gareth Jones, Javier Cirre and Pablo Martín for many helpful ideas.

\section{$\S 1$ Belyi's Theorem for curves over non algebraically closed base fields}

In this section we extend Belyi's theorem from complex curves to curves over an arbitrary subfield of $\mathbb{C}$. We follow the approach used in $[\mathrm{Kö}]$ and based on earlier work by Wolfart, see [Wo]. Related results may be found in the recent paper [Go] by González-Diez.

We fix a subfield $C$ of $\mathbb{C}$. In this section, by a curve over $C$ we mean a smooth projective geometrically connected variety of dimension 1 over $C$. We recall that mapping a curve $X$ over $C$ to its function field $K(X)$ yields an antiequivalence between the category of curves over $C$ and non-constant morphisms on the one hand and the category of finitely generated field extensions $K$ of $C$ of transcendence degree 1 such that $C$ is algebraically closed in $K$ on the other hand. Furthermore we will frequently use the well-known fact that elements of $K(X)$ correspond to morphisms $X \rightarrow \mathbb{P}_{C}^{1}$ of varieties over $C$. As usual we say that a curve $X$ over $C$ can be defined over a subfield $D$ of $C$ if there is a curve $Y$ over $D$ such that $Y_{C}:=Y \times_{D} C$ 
is isomorphic to $X$ over $C$. This is obviously equivalent to the condition that there is a subfield $K$ of the function field $K(X)$ containing $D$ which is finitely generated and of transcendence degree 1 over $D$ such that $D$ is algebraically closed in $K$ and such that the canonical homomorphism

$$
\operatorname{Quot}\left(C \otimes_{D} K\right) \rightarrow K(X)
$$

from the field of fractions $\operatorname{Quot}\left(C \otimes_{D} K\right)$ of $C \otimes_{D} K$ to $K(X)$ is bijective. Note that $C \otimes_{D} K$ is an integral domain by the following lemma.

(1.1) Lemma. Let $D$ be any field of characteristic 0 and let $K$ be any extension field of $D$. Then $D$ is algebraically closed in $K$ if and only if, for every extension field $C$ of $D$, the tensor product $C \otimes_{D} K$ is an integral domain. In this case, $C$ is algebraically closed in $\operatorname{Quot}\left(C \otimes_{D} K\right)$.

Proof. See Proposition (4.3.2) and Proposition (4.3.5) in [EGAIV].

We recall (see $\S 2$ in [Kö]) that the moduli field $M(X, t)$ of a finite morphism $t: X \rightarrow$ $\mathbb{P}_{\mathbb{C}}^{1}$ from a curve $X$ over $\mathbb{C}$ to the complex projective line $\mathbb{P}_{\mathbb{C}}^{1}$ is defined to be the subfield of $\mathbb{C}$ fixed by the subgroup $U(X, t)$ of Aut $(\mathbb{C})$ consisting of all automorphisms $\sigma$ of $\mathbb{C}$ such that there is an isomorphism $f_{\sigma}: X^{\sigma} \rightarrow X$ of varieties over $\mathbb{C}$ such that the following diagram commutes:

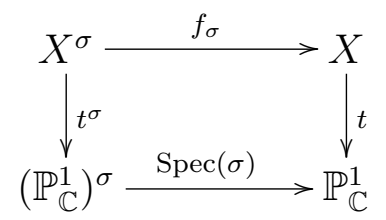

Here, the notation $X^{\sigma}$ means the scheme $X$ viewed as a variety over $\mathbb{C}$ via the new structure morphism $X \stackrel{p}{\rightarrow} \operatorname{Spec}(\mathbb{C}) \stackrel{\operatorname{Spec}(\sigma)}{\longrightarrow} \operatorname{Spec}(\mathbb{C})$ where $p$ denotes the structure morphism of the given variety $X$ over $\mathbb{C}$ (see $\S 1$ in $[\mathrm{Kö}]$ for further explanations of this notation).

The following proposition is a special case of Corollary (3.2) in [Kö].

(1.2) Proposition. If the critical values of $t$ are $\mathbb{Q}$-rational then the moduli field $M(X, t)$ is a number field.

Let now $t: X \rightarrow \mathbb{P}_{C}^{1}$ be a finite morphism from a curve $X$ over the given subfield $C$ of $\mathbb{C}$ to the projective line $\mathbb{P}_{C}^{1}$. Then the subgroup $U\left(X_{\mathbb{C}}, t_{\mathbb{C}}\right)$ of Aut $(\mathbb{C})$ obviously contains $\operatorname{Aut}(\mathbb{C} / C)$. Thus the moduli field $M\left(X_{\mathbb{C}}, t_{\mathbb{C}}\right)$ of $t_{\mathbb{C}}: X_{\mathbb{C}} \rightarrow \mathbb{P}_{\mathbb{C}}^{1}$ is contained in $\mathbb{C}^{\operatorname{Aut}(\mathbb{C} / C)}=C$ (by Lemma (1.4) in $\left.[\mathrm{Kö}]\right)$. 
(1.3) Proposition. If there is a C-rational unramified point $P$ on $X$ such that $Q:=t(P)$ lies in $\mathbb{Q}$ then the curve $X / C$ and the morphism $t$ can be defined over a finite extension of the moduli field $M\left(X_{\mathbb{C}}, t_{\mathbb{C}}\right)$ (inside $C$ ).

Note that Proposition (1.3) is stronger than Theorem (2.2) in [Kö] which only implies that $X_{\mathbb{C}} / \mathbb{C}\left(\right.$ or $\left.X_{\bar{C}} / \bar{C}\right)$ is defined over a finite extension of $M\left(X_{\mathbb{C}}, t_{\mathbb{C}}\right)$. For the convenience of the reader we give the details of the proof of Proposition (1.3) which is quite similar to the proof of Theorem (2.2) in [Kö].

Proof. By the Riemann-Roch Theorem (see [Mo]) applied to the divisor $D:=$ (genus $(X)+1)[P]$ there is a non-constant meromorphic function $z$ on $X$ (defined over $C$ ) such that $P$ is the only pole of $z$. Then the function field $K(X)$ of $X$ is generated over $C$ by $t$ and $z$. This follows from the easy observation that the field extension $K(X) / C(t, z)$ is a subextension of $K(X) / C(t)$ and of $K(X) / C(z)$ and that, hence, the corresponding morphism of curves over $C$ is both unramified and totally ramified at $P$. We assume furthermore that we have chosen $z$ in such a way that the pole order $m:=-\operatorname{ord}_{P}(z) \in \mathbb{N}$ is minimal. Then the constant function 1 and the function $z$ form a basis of the $C$-vector space

$$
V:=\left\{x \in K(X): x \text { has a pole at most at } P \text { and } \operatorname{ord}_{P}(x) \geq-m\right\} .
$$

To see this, let $x \in V$; if $x$ is not a constant we have $\operatorname{ord}_{P}(x)=-m \stackrel{\text { def }}{=} \operatorname{ord}_{P}(z)$ since $m$ was minimal; hence there is a constant $\alpha \in C$ such that $-\operatorname{ord}_{P}(x-\alpha z)<m$; thus, again since $m$ was minimal, the function $x-\alpha z$ is constant, as desired.

Since $t$ is unramified at $P$, the meromorphic function $t-Q$ on $X$ is a local parameter on $X$ at $P$. Obviously, there is a unique function $z^{\prime} \in V$ such that the leading coefficient (i.e., the coefficient of $\left.(t-Q)^{-m}\right)$ and the constant coefficient (i.e., the coefficient of $\left.(t-Q)^{0}\right)$ in the Laurent expansion of $z^{\prime}$ with respect to the local parameter $t-Q$ are 1 and 0 , respectively. We may and we will assume that $z=z^{\prime}$.

We now claim that the minimal polynomial of $z$ over $C(t)$ has coefficients in $k(t)$ where $k$ is a finite extension of $M\left(X_{\mathbb{C}} / t_{\mathbb{C}}\right)$ inside $C$. Then the field extension $K(X) / C(t)$ is defined over $k$. This means that $X / C$ and the morphism $t$ are defined over $k$, as was to be shown.

To prove the above claim we introduce the notation $U\left(X_{\mathbb{C}}, t_{\mathbb{C}}, P\right)$ for the subgroup of $U\left(X_{\mathbb{C}}, t_{\mathbb{C}}\right)$ consisting of all automorphisms $\sigma$ of $\mathbb{C}$ such that there is an isomorphism $f_{\sigma}: X_{\mathbb{C}}^{\sigma} \rightarrow X_{\mathbb{C}}$ of varieties over $\mathbb{C}$ such that the diagram

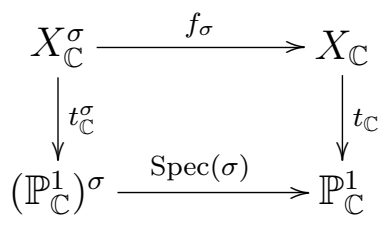


commutes and such that $f_{\sigma}\left(P^{\sigma}\right)=P$; here, $P^{\sigma}$ denotes the point in $X_{\mathbb{C}}^{\sigma}$ corresponding to $P$. Note that $f_{\sigma}$ is unique since $\operatorname{Aut}\left(t_{\mathbb{C}}\right)$ acts freely on the fibre $t_{\mathbb{C}}^{-1}(Q)$. Thus mapping $\sigma$ to the automorphism of the function field $K\left(X_{\mathbb{C}}\right)$ induced by $f_{\sigma}$ yields an action of $U\left(X_{\mathbb{C}}, t_{\mathbb{C}}, P\right)$ on $K\left(X_{\mathbb{C}}\right)$ by semilinear field automorphisms which fix $t_{\mathbb{C}} \in K\left(X_{\mathbb{C}}\right)$. The meromorphic function $z_{\mathbb{C}} \in K\left(X_{\mathbb{C}}\right)$ is invariant under the action of $U\left(X_{\mathbb{C}}, t_{\mathbb{C}}, P\right)$ defined above since the image of $z_{\mathbb{C}}$ under $\sigma \in U\left(X_{\mathbb{C}}, t_{\mathbb{C}}, P\right)$ has the same three defining properties as $z_{\mathbb{C}}$, as one easily checks. Hence the minimal polynomial of $z_{\mathbb{C}}$ over $\mathbb{C}\left(t_{\mathbb{C}}\right)$ is invariant under the action of $U\left(X_{\mathbb{C}}, t_{\mathbb{C}}, P\right)$, i.e. it has coefficients in $k\left(t_{\mathbb{C}}\right)$ where $k$ is the subfield of $\mathbb{C}$ fixed by $U\left(X_{\mathbb{C}}, t_{\mathbb{C}}, P\right)$. We obviously have $\operatorname{Aut}(\mathbb{C} / C) \subseteq U\left(X_{\mathbb{C}}, t_{\mathbb{C}}, P\right)$ since $P$ is $C$-rational. Thus $k$ is contained in $\mathbb{C}^{\operatorname{Aut}(\mathbb{C} / C)}=C$ (by Lemma $(1.4)$ in $[\mathrm{Kö}]$ ). Finally, being the stabilizer of $[P]$ under the (well-defined!) action $(\sigma,[P]) \mapsto\left[f_{\sigma}(P)\right]$ of $U\left(X_{\mathbb{C}}, t_{\mathbb{C}}\right)$ on $t_{\mathbb{C}}^{-1}(Q) / \operatorname{Aut}\left(t_{\mathbb{C}}\right)$, the subgroup $U\left(X_{\mathbb{C}}, t_{\mathbb{C}}, P\right)$ has finite index in $U\left(X_{\mathbb{C}}, t_{\mathbb{C}}\right)$. Hence, by Lemma (1.6) in [Kö], $k$ is a finite extension of $M\left(X_{\mathbb{C}}, t_{\mathbb{C}}\right)$, as was to be shown.

(1.4) Theorem (Belyi's theorem for curves over a non algebraically closed base field). Lex $X$ be a curve over $C$.

(a) If $X$ can be defined over $\overline{\mathbb{Q}} \cap C$, then there exists a finite morphism $t: X \rightarrow \mathbb{P}_{C}^{1}$ (defined over $C$ !) such that the critical values of $t_{\mathbb{C}}: X_{\mathbb{C}} \rightarrow \mathbb{P}_{\mathbb{C}}^{1}$ lie in $\{0,1, \infty\}$. (The morphism $t$ may actually be chosen to be defined even over $\overline{\mathbb{Q}} \cap C$.)

(b) The converse of statement (a) is true if there exists an unramified $C$-rational point $P$ on $X$ such that $(P)$ lies in $\mathbb{Q}$.

Proof.

(a) Let $X_{\overline{\mathbb{Q}} \cap C}$ be a model of $X$ over $\overline{\mathbb{Q}} \cap C$ and let $t^{\prime}: X_{\overline{\mathbb{Q}} \cap C} \rightarrow \mathbb{P}_{\overline{\mathbb{Q}} \cap C}^{1}$ be any finite morphism. By Lemma (3.4) in [Kö], the critical values of $t_{\mathbb{C}}^{\prime}: X_{\mathbb{C}} \rightarrow \mathbb{P}_{\mathbb{C}}^{1}$ lie in $\overline{\mathbb{Q}} \cup\{\infty\}$. By Lemma (3.5) and (the proof of) Lemma (3.6) in [Kö] there is a finite morphism $r: \mathbb{P}_{\mathbb{C}}^{1} \rightarrow \mathbb{P}_{\mathbb{C}}^{1}$ defined over $\mathbb{Q}$ such that the critical values of $r$ and the images of the critical values of $t_{\mathbb{C}}^{\prime}$ under $r$ lie in $\{0,1, \infty\}$. We write $r$ also for the corresponding morphism $r: \mathbb{P}_{\overline{\mathbb{Q}} \cap C}^{1} \rightarrow \mathbb{P}_{\overline{\mathbb{Q}} n C}^{1}$. Let $t$ denote the composition $r \circ t^{\prime}$. Then $t_{C}: X \rightarrow \mathbb{P}_{C}^{1}$ is a finite morphism defined over $\overline{\mathbb{Q}} \cap C$ such that the critical values of $t_{\mathbb{C}}$ lie in $\{0,1, \infty\}$.

(b) By Proposition (1.2), the moduli field $M\left(X_{\mathbb{C}}, t_{\mathbb{C}}\right)$ of $t_{\mathbb{C}}$ is a number field (contained in $C$ ). By Proposition (1.3), the curve $X$ and the morphism $t: X \rightarrow \mathbb{P}_{\mathbb{C}}^{1}$ can be defined over a finite extension of $M\left(X_{\mathbb{C}}, t_{\mathbb{C}}\right.$ ) (inside $C$ ). Thus $X$ and $t$ can be defined over $\overline{\mathbb{Q}} \cap C$, as was to be shown. 


\section{$\S 2$ Belyi's Theorem for Klein surfaces}

In this section we develop a 'real' Belyi theory; its highlight is a Belyi theorem for Klein surfaces, see Theorem (2.6).

We recall that the canonical functor from the category of smooth projective curves over $\mathbb{C}$ to the category of compact Riemann surfaces is an equivalence of categories. In particular, every compact connected Riemann surface $X$ is isomorphic to the Riemann surface associated with a smooth projective connected curve over $\mathbb{C}$ and the field $M_{\mathrm{RS}}(X)$ of meromorphic functions on $X$ can be identified with the function field of this curve over $\mathbb{C}$.

As usual we say that a compact Riemann surface $X$ can be defined over a subfield $C$ of $\mathbb{C}$ if the corresponding smooth projective connected curve over $\mathbb{C}$ can be defined over $C$. A reader not familiar with the language of schemes may prefer to work with the following characterization. By $\S 1$ and the remarks after Definition (4.5.2) in [EGAIV], the Riemann surface $X$ can be defined over $C$ if and only if there exists a subfield $K$ of $M_{\mathrm{RS}}(X)$ containing $C$ which is finitely generated and of transcendence degree 1 over $C$ such that the canonical map $\mathbb{C} \otimes_{C} K \rightarrow M_{\mathrm{RS}}(X)$ is injective and $M_{\mathrm{RS}}(X)$ is the field of fractions of $\mathbb{C} \otimes_{C} K$ via this map.

Let now $S$ be a compact connected Klein surface. We refer the reader to [AG] and $[\mathrm{Ga}]$ for basic facts about Klein surfaces. We denote the field of meromorphic functions of $S$ by $M_{\mathrm{KS}}(S)$. By Theorem 1.4.6 in [AG], this is the set of morphisms (of Klein surfaces) from $S$ to the compactified closed upper half plane

$$
\Delta:=\{z \in \mathbb{C}: \operatorname{Im}(z) \geq 0\} \cup\{\infty\} .
$$

$M_{\mathrm{KS}}(S)$ is a finitely generated extension of $\mathbb{R}$ of transcendence degree 1 .

(2.1) Definition. We say that $S$ can be defined over the field $\overline{\mathbb{Q}} \cap \mathbb{R}$ of real algebraic numbers if there exists a subfield $K$ of $M_{\mathrm{KS}}(S)$ containing $\overline{\mathbb{Q}} \cap \mathbb{R}$ which is finitely generated and of transcendence degree 1 over $\overline{\mathbb{Q}} \cap \mathbb{R}$ such that the canonical map $\mathbb{R} \otimes_{\overline{\mathbb{Q}} \cap \mathbb{R}} K \rightarrow M_{\mathrm{KS}}(S)$ is injective and $M_{\mathrm{KS}}(S)$ is the field of fractions of $\mathbb{R} \otimes_{\overline{\mathbb{Q}} \cap \mathbb{R}} K$ via this map.

Note that $S$ can obviously be defined over $\overline{\mathbb{Q}} \cap \mathbb{R}$ if and only if the projective real algebraic curve corresponding to $M_{\mathrm{KS}}(S)$ can be defined over $\overline{\mathbb{Q}} \cap \mathbb{R}$. When using this characterization, a real projective algebraic curve has basically to be understood as a scheme, i.e. as a space together with the defining equations. In contrast to complex curves, it is not sufficient to think of a real curve just as the set of real points, nor as the set of its complex points. In order to make this section (and this 
paper) accessible to as wide an audience as possible we have decided to work with the language of function fields.

(2.2) Lemma. Let $X$ be a compact connected Riemann surface. Then $X$ can be defined over $\overline{\mathbb{Q}}$ (as Riemann surface) if and only if $X$, viewed as Klein surface, can be defined over $\overline{\mathbb{Q}} \cap \mathbb{R}$.

Proof. We first prove the only-if-part. Let $K$ be a subfield of $M_{\mathrm{RS}}(X)$ containing $\overline{\mathbb{Q}}$ which is finitely generated and of transcendence degree 1 over $\overline{\mathbb{Q}}$ such that the canonical map $\mathbb{C} \otimes_{\overline{\mathbb{Q}}} K \rightarrow M_{\mathrm{RS}}(X)$ is injective and $M_{\mathrm{RS}}(X)$ is the field of fractions of $\mathbb{C} \otimes_{\overline{\mathbb{Q}}} K$. The canonical $\mathbb{R}$-algebra homomorphism $\mathbb{R} \otimes_{\overline{\mathbb{Q}} \cap \mathbb{R}} \overline{\mathbb{Q}} \rightarrow \mathbb{C}$ is bijective since it takes the $\mathbb{R}$-basis $1 \otimes 1,1 \otimes i$ of $\mathbb{R} \otimes_{\overline{\mathbb{Q}} \cap \mathbb{R}} \overline{\mathbb{Q}}$ to the $\mathbb{R}$-basis $1, i$ of $\mathbb{C}$. Hence we obtain

$$
\mathbb{R} \otimes_{\overline{\mathbb{Q}} \cap \mathbb{R}} K \cong \mathbb{R} \otimes_{\overline{\mathbb{Q}} \cap \mathbb{R}} \overline{\mathbb{Q}} \otimes_{\overline{\mathbb{Q}}} K \cong \mathbb{C} \otimes_{\overline{\mathbb{Q}}} K
$$

and

$$
\operatorname{Quot}\left(\mathbb{R} \otimes_{\overline{\mathbb{Q}} \cap \mathbb{R}} K\right) \cong \operatorname{Quot}\left(\mathbb{C} \otimes_{\overline{\mathbb{Q}}} K\right) \cong M_{\mathrm{RS}}(X) \cong M_{\mathrm{KS}}(X),
$$

as desired.

We now assume that $X$, viewed as Klein surface, can be defined over $\overline{\mathbb{Q}} \cap \mathbb{R}$. Let $K$ be a subfield of $M_{\mathrm{KS}}(X)$ containing $\overline{\mathbb{Q}} \cap \mathbb{R}$ which is finitely generated and of transcendence degree 1 over $\overline{\mathbb{Q}} \cap \mathbb{R}$ such that the canonical map $\mathbb{R} \otimes_{\overline{\mathbb{Q}} \cap \mathbb{R}} K \rightarrow M_{\mathrm{KS}}(X)$ is injective and such that $M_{\mathrm{KS}}(X)$ is the field of fractions of $\mathbb{R} \otimes_{\overline{\mathbb{Q}} \cap \mathbb{R}} K$. We have $i=\sqrt{-1} \in K$ because otherwise the ring

$$
\mathbb{R} \otimes_{\overline{\mathbb{Q}} \cap \mathbb{R}} K \otimes_{\mathbb{Q}} \mathbb{Q}[i] \cong \mathbb{R} \otimes_{\overline{\mathbb{Q}} \cap \mathbb{R}} K[i] \cong \mathbb{R} \otimes_{\overline{\mathbb{Q}} \cap \mathbb{R}} \overline{\mathbb{Q}} \otimes_{\overline{\mathbb{Q}}} K[i] \cong \mathbb{C} \otimes_{\overline{\mathbb{Q}}} K[i]
$$

would be an integral domain by Lemma (1.1); but the canonical map

$$
\operatorname{Quot}\left(\mathbb{R} \otimes_{\overline{\mathbb{Q}} \cap \mathbb{R}} K\right) \otimes_{\mathbb{Q}} \mathbb{Q}[i] \rightarrow \operatorname{Quot}\left(\mathbb{R} \otimes_{\overline{\mathbb{Q}} \cap \mathbb{R}} K \otimes_{\mathbb{Q}} \mathbb{Q}[i]\right)
$$

is injective (We may use the basis $1, i$ of $\mathbb{Q}[i]$ over $\mathbb{Q}$ to check this.) and the ring $\operatorname{Quot}\left(\mathbb{R} \otimes_{\overline{\mathbb{Q}} \cap \mathbb{R}} K\right) \otimes_{\mathbb{Q}} \mathbb{Q}[i] \cong M_{\mathrm{KS}}(X) \otimes_{\mathbb{Q}} \mathbb{Q}[i] \cong M_{\mathrm{RS}}(X) \otimes_{\mathbb{Q}} \mathbb{Q}[i] \cong M_{\mathrm{RS}}(X) \times M_{\mathrm{RS}}(X)$ is not an integral domain. Hence $K$ contains $\overline{\mathbb{Q}}=(\overline{\mathbb{Q}} \cap \mathbb{R})[i]$ and we have

$$
\operatorname{Quot}\left(\mathbb{C} \otimes_{\overline{\mathbb{Q}}} K\right) \cong \operatorname{Quot}\left(\mathbb{R} \otimes_{\overline{\mathbb{Q}} \cap \mathbb{R}} K\right) \cong M_{\mathrm{KS}}(X) \cong M_{\mathrm{RS}}(X),
$$

as desired.

We recall that the canonical functor from the category of Riemann surfaces to the category of Klein surfaces admits a right adjoint functor. In other words, for every 
Klein surface $S$ there exists a Riemann surface $S^{\mathrm{c}}$ together with a morphism $f_{S}$ : $S^{\text {c }} \rightarrow S$ of Klein surfaces such that for every Riemann surface $X$ the map

$$
\operatorname{Mor}_{\mathrm{RS}}\left(X, S^{\mathrm{c}}\right) \rightarrow \operatorname{Mor}_{\mathrm{KS}}(X, S), \quad \rho \mapsto f_{S} \circ \rho,
$$

is bijective (see Proposition 1.6.2 in $[\mathrm{AG}]$ ). In fact the map $f_{S}$ is a double cover; we therefore call $S^{\text {c }}$ the complex double of $S$. The complex double $S^{\text {c }}$ comes with an antiholomorphic involution $\tau$ such that $S^{\mathrm{c}} /\langle\tau\rangle \cong S$. In fact this gives an equivalence between the category of Klein surfaces and the category of pairs consisting of a Riemann surface and an antiholomorphic involution. For instance, $\Delta^{\mathrm{c}}$ is the Riemann sphere $\hat{\mathbb{C}}$ and $f_{\Delta}: \Delta^{\mathrm{c}} \rightarrow \Delta$ is the folding map $\hat{\mathbb{C}} \rightarrow \Delta, a+b i \mapsto a+|b| i, \infty \mapsto \infty$; or, if $S$ is the Klein surface associated with a Riemann surface $X$, then $S^{\mathrm{c}}$ is the disjoint union of the Riemann surfaces $X$ and $\bar{X}$ (where $\bar{X}$ denotes the topological space $X$ equipped with the antiholomorphic structure) and $f_{S}: X \cup \bar{X} \rightarrow S$ is the identity map on both $X$ and $\bar{X}$.

(2.3) Definition. A morphism $\beta: S \rightarrow \Delta$ from a compact Klein surface $S$ to the compactified upper half plane $\Delta$ is called a Belyi map on $S$ if the complex double $\beta^{\mathrm{c}}: S^{\mathrm{c}} \rightarrow \Delta^{\mathrm{c}} \cong \hat{\mathbb{C}}$ of $\beta$ is a Belyi map on the Riemann surface $S^{\mathrm{c}}$, i.e. if the restriction of $\beta^{\mathrm{c}}$ to every connected component of $S^{\mathrm{c}}$ has at most 3 critical values. We shall also call $\beta$ a real Belyi function.

(2.4) Lemma. Let $X$ be a compact connected Riemann surface. Then $X$ (as Riemann surface) admits a Belyi map from $X$ to $\hat{\mathbb{C}}$ if and only if $X$, viewed as Klein surface, admits a Belyi map from $X$ to $\Delta$.

Proof. We first prove the if-part. Let $\beta: X \rightarrow \Delta$ be a Belyi map on the Klein surface $X$. Then, by definition, the induced morphism $\beta^{\mathrm{c}}: X^{\mathrm{c}} \rightarrow \Delta^{\mathrm{c}} \cong \hat{\mathbb{C}}$ is a Belyi map on the Riemann surface $X^{\mathrm{c}}$. In particular, the restriction of $\beta^{\mathrm{c}}$ to the connected component $X$ of $X^{\mathrm{c}}=X \cup \bar{X}$ is a Belyi map on the given Riemann surface $X$, as desired.

We now prove the only-if-part. Let $\beta: X \rightarrow \hat{\mathbb{C}}$ be a Belyi map on the Riemann surface $X$. Then the composition $X \stackrel{\beta}{\rightarrow} \hat{\mathbb{C}} \stackrel{\rho}{\rightarrow} \Delta$ of $\beta$ with the folding map $\rho: \hat{\mathbb{C}} \rightarrow \Delta$ is a Belyi map on the Klein surface $X$ since the induced morphism $(\rho \circ \beta)^{\mathrm{c}}: X \cup \bar{X}=X^{\mathrm{c}} \rightarrow \Delta^{\mathrm{c}} \cong \hat{\mathbb{C}}$ is equal to $\beta$ on $X$ and equal to the complex conjugate $\bar{\beta}$ of $\beta$ on $\bar{X}$ and, hence, is a Belyi map on the Riemann surface $X^{\mathrm{c}}$.

(2.5) Lemma. If a compact Klein surface $S$ admits a Belyi map $\beta: S \rightarrow \Delta$ then it also admits a Belyi map $\gamma: S \rightarrow \Delta$ such that the critical values of $\gamma^{\mathrm{c}}: S^{\mathrm{c}} \rightarrow \hat{\mathbb{C}}$ lie in $\{0,1, \infty\}$.

Proof. We may assume that $S$ is connected. 
We first consider the case that the critical values of $\beta^{\text {c }}: S^{\text {c }} \rightarrow \hat{\mathbb{C}}$ lie in $\mathbb{R} \cup\{\infty\}$. Then we can find a Möbius transformation $r: \hat{\mathbb{C}} \rightarrow \widehat{\mathbb{C}}$ with real coefficients which maps the critical values of $\beta^{\mathrm{c}}$ into $\{0,1, \infty\}$. In particular, the unique $\gamma \in M_{\mathrm{KS}}(S)$ such that $\gamma^{\mathrm{c}}=r \circ \beta^{\mathrm{c}}$ is a Belyi map on $S$ of the desired type.

If a critical value of $\beta^{\mathrm{c}}$, say $P$, lies in $\mathbb{C} \backslash \mathbb{R}$ then also its complex conjugate $\bar{P}$ is a critical value of $\beta^{\mathrm{c}}$ since $\beta^{\mathrm{c}}$ is defined over $\mathbb{R}$. By the same argument, the third one (if there is one), say $Q$, must lie in $\mathbb{R} \cup\{\infty\}$. As above, by composing with a Möbius transformation with real coefficients, we may assume that $Q=\infty$. Let $a:=-2 \operatorname{Re}(P) \in \mathbb{R}$ and $b:=|P|^{2} \in \mathbb{R}$. Then the real quadratic polynomial $r:=$ $X^{2}+a X+b$ takes the three critical values $P, \bar{P}, Q=\infty$ of $\beta^{\mathrm{c}}$ to the two values $0, \infty$ and it has the two critical values $b-\frac{a^{2}}{4}$ and $\infty$. Thus the unique $\tilde{\beta} \in M_{\mathrm{KS}}(S)$ such that $\tilde{\beta}^{\mathrm{c}}=r \circ \beta^{\mathrm{c}}$ is a Belyi map on $S$ of the type considered in the first case. Therefore $S$ admits a Belyi map of the desired type also in the general case.

(2.6) Theorem (Belyi's Theorem for Klein surfaces). Let $S$ be a compact connected Klein surface. If $S$ can be defined over $\overline{\mathbb{Q}} \cap \mathbb{R}$ then $S$ admits a Belyi map $\beta: S \rightarrow \Delta$. If the boundary $\partial S$ of $S$ is non-empty or if $S$ is the Klein surface associated with a compact connected Riemann surface then the converse is true as well.

Proof. If $S$ is the Klein surface associated with a Riemann surface then Theorem (2.6) follows from Lemma (2.2), Lemma (2.4) and Belyi's Theorem for Riemann surfaces (see Theorem (3.3) in [Kö]).

Hence we may assume that $S$ is not the Klein surface associated with a Riemann surface. Then its complex double $S^{\mathrm{c}}$ is connected by Lemma 1.6.3 in [AG] and Remark (1.5)(ii) in [Ga]; in particular $M_{\mathrm{RS}}\left(S^{\mathrm{c}}\right)$ is a field and we have $M_{\mathrm{RS}}\left(S^{\mathrm{c}}\right)=$ $M_{\mathrm{KS}}(S) \otimes_{\mathbb{Q}} \mathbb{Q}[i]$ by Corollary 1.6.5 in $[\mathrm{AG}]$. In other words, the smooth projective curve $X$ over $\mathbb{R}$ corresponding to the function field $M_{\mathrm{KS}}(S)$ over $\mathbb{R}$ is geometrically connected, and we may apply the results of $\S 1$.

If $S$ can be defined over $\overline{\mathbb{Q}} \cap \mathbb{R}$ then obviously the curve $X$ can be defined over $\overline{\mathbb{Q}} \cap \mathbb{R}$. Hence, by Theorem (1.4)(a), there exists a finite morphism $t: X \rightarrow \mathbb{P}_{\mathbb{R}}^{1}$ (defined over $\mathbb{R})$ such that the critical values of $t_{\mathbb{C}}: X_{\mathbb{C}} \rightarrow \mathbb{P}_{\mathbb{C}}^{1}$ lie in $\{0,1, \infty\}$. Thus the corresponding morphism $\beta: S \rightarrow \Delta$ is a Belyi map on the Klein surface $S$, as desired.

We now assume that $S$ admits a Belyi map $\beta: S \rightarrow \Delta$ and that $\partial S \neq \emptyset$. As $\partial S$ is homoemorphic to the real manifold $X(\mathbb{R})$ of $\mathbb{R}$-valued points on $X$ (see Theorem (4.17) in $[\mathrm{Ga}])$ there exists an $\mathbb{R}$-valued point on $X$, say $P_{0}$. Since the manifold $X(\mathbb{R})$ is of dimension 1 there exists a neighborhood $U$ of $P_{0}$ in $X(\mathbb{R})$ homeomorphic to an interval in $\mathbb{R}$. The morphism $t: X \rightarrow \mathbb{P}_{\mathbb{R}}^{1}$ corresponding to the given Belyi map $\beta: S \rightarrow \Delta$ maps $U$ to an interval in $\mathbb{R} \cup\{\infty\}$. In particular there are infinitely many 
points $P$ in $U \subseteq X(\mathbb{R})$ such that $t(P)$ lies in $\mathbb{Q}$. Since the set of ramified points is finite, there hence exists an unramified $\mathbb{R}$-rational point $P$ on $X$ such that $t(P)$ lies in $\mathbb{Q}$. Thus, by Theorem (1.4)(b), the curve $X$ and hence the Klein surface $S$ can be defined over $\overline{\mathbb{Q}} \cap \mathbb{R}$, as was to be shown.

(2.7) Question. Let $S$ be a compact connected Klein surface which admits a Belyi map. By Theorem (2.6), the Klein surface $S$ can be defined over $\overline{\mathbb{Q}} \cap \mathbb{R}$ if $\partial S \neq \emptyset$ or if $(\partial S=\emptyset$ and $S$ is orientable). Does the same conclusion hold also in the remaining case that $\partial S=\emptyset$ and $S$ is not orientable?

(2.8) Example. Let $S$ be a compact connected Klein surface which is non-orientable or has non-empty boundary. Then the complex double $S^{\mathrm{c}}$ is connected again. Let $g$ denote the genus of the compact Riemann surface $S^{\text {c }}$. In this example we want to show that Question (2.7) has an affirmative answer if $g=0$ or $g=1$.

(a) Let $g=0$, i.e. the Riemann surface $S^{\text {c }}$ is isomorphic to the Riemann sphere $\hat{\mathbb{C}}$. Then, according to Theorem 1.9.4 in [AG], the Klein surface $S$ is isomorphic to either the compactified upper halfplane $\Delta$ or to the real projective plane $\mathbb{P}^{2}(\mathbb{R})$ which, as Klein surface, is defined as $\hat{\mathbb{C}} /\langle\sigma\rangle$ where the antiholomorphic involution $\sigma$ sends $z \in \hat{\mathbb{C}}$ to $-\frac{1}{\bar{z}}$. In the first case, the function field of $S$ is given by the field $\mathbb{R}(z)$ of rational functions in one variable. In the second case, the function field of $S$ is isomorphic to the field of fractions of the integral domain $\mathbb{R}[X, Y] /\left(X^{2}+Y^{2}+1\right)$ embedded into the function field $\mathbb{C}(z)$ of the Riemann sphere via $X \mapsto \frac{1}{2}\left(z-\frac{1}{z}\right)$ and $Y \mapsto \frac{1}{2 i}\left(z+\frac{1}{z}\right)$. Both of these Klein surfaces are obviously defined over $\mathbb{Q}$, the smallest field of characteristic 0. In particular, Question (2.7) has an affirmative answer if $S$ is the real projective plane.

(b) Let $g=1$, i.e. the Riemann surface $S^{\text {c }}$ is an elliptic curve. From $\S 9$ in [AG] we know $S$ that must be homoemorphic to either an annulus, a Möbius strip or to a Klein bottle. By Example 1.7 in [Kö], the moduli field of $S^{\mathrm{c}}$ is $\mathbb{Q}(j)$ where $j \in \mathbb{R}$ denotes the $j$-invariant of $S^{\text {c }}$. According to Chapters 14.34, 14.41 and 14.42 in [Al], the function field of $S$ is isomorphic to the field of fractions of an integral domain of the form $\mathbb{R}[X, Y] /\left(Y^{2} \pm\left(1 \pm X^{2}\right)\left(1 \pm \lambda X^{2}\right)\right)$ where $\lambda \in \mathbb{R}$ is a/the Legendre modulus of the elliptic curve $S^{\text {c }}$. Here $X$ corresponds to (a constant multiple of) the Jacobi elliptic function sn ('sinam function') and $Y$ to its derivative. As $\lambda$ satisfies the equation

$$
j=k \frac{\left(\lambda^{2}-\lambda+1\right)^{3}}{\lambda^{2}(\lambda-1)^{2}} \quad \text { with some } k \in \mathbb{Q}
$$

(see Example 1 on p. 566 in [JS2]) we see that $S$ can be defined over a (real) extension field of $\mathbb{Q}(j)$ of degree at most 6. Now Proposition (1.2) implies that Question (2.7) has an affirmative answer also if $S$ is homeomorphic to the Klein bottle. 
(2.9) Example. Let $S$ and $j$ be as in Example (2.8)(b) above. We now want to show that $S$ can be defined not only over a finite extension of $\mathbb{Q}(j)$ but over $\mathbb{Q}(j)$ itself if $S$ is homeomorphic to an annulus or to a Möbius strip. Whether the same holds true if $S$ is homeomorphic to a Klein bottle remains open.

We consider the two real elliptic curves $X_{+}(j)$ and $X_{-}(j)$ given by the following equations defined over $\mathbb{Q}(j)$ :

$$
\begin{array}{ccc}
X_{+}(j): \quad y^{2}=4 x^{3}-\frac{27 j}{j-1} x-\frac{27 j}{j-1} & \text { if } j \neq 0,1 \\
y^{2}=4 x^{3}-1 & \text { if } j=0 \\
y^{2}=4 x^{3}-x & \text { if } j=1 \\
X_{-}(j): \quad y^{2}=4 x^{3}-\frac{27 j}{j-1} x+\frac{27 j}{j-1} & \text { if } j \neq 0,1 \\
& y^{2}=4 x^{3}+1 & \text { if } j=0 \\
y^{2}=4 x^{3}+x & \text { if } j=1
\end{array}
$$

It is easy to see that the $j$-invariant of both $X_{+}(j)$ and $X_{-}(j)$ is $j$. However $X_{+}(j)$ is not $\mathbb{R}$-isomorphic to $X_{-}(j)$. To see this we recall that two real elliptic curves $X$ and $\tilde{X}$ given by the equations $y^{2}=4 x^{3}-g_{2} x-g_{3}$ and $y^{2}=4 x^{3}-\tilde{g}_{2} x-\tilde{g}_{3}$ are $\mathbb{R}$-isomorphic if and only if there exists a $u \in \mathbb{R}$ such that the second equation is obtained from the first equation by the substitutions $x \mapsto u^{2} x$ and $y \mapsto u^{3} y$. In particular, if $X$ and $\tilde{X}$ are isomorphic then the sign of $g_{2}$ is equal to the sign of $\tilde{g}_{2}$ and the sign of $g_{3}$ is equal to the sign of $\tilde{g}_{3}$. As this is not true for $X_{+}(j)$ and $X_{-}(j)$, they are not $\mathbb{R}$-isomorphic. Obviously, both $X_{+}(j)$ and $X_{-}(j)$ have $\mathbb{R}$-valued points; hence the corresponding Klein surfaces $S_{+}(j)$ and $S_{-}(j)$ have non-empty boundary and are therefore not homeomorphic to a Klein bottle. From the classification of Klein surfaces of genus 1 (see $\S 9$ of Chapter 1 in $[A G]$ ) we know that there are exactly two isomorphism classes of connected compact Klein surfaces which are not homeomorphic to a Klein bottle and whose complex double is connected and has genus 1 and $j$-invariant $j$. Hence $S$ is isomorphic to $S_{+}(j)$ or $S_{-}(j)$ and therefore defined over $\mathbb{Q}(j)$.

\section{$\S 3$ Belyi Functions on Klein Surfaces and the Ex- tended Modular Group}

The object of this section is to extend various characterizations of Belyi surfaces (see Section 4 in [JS2]) from Riemann surfaces to Klein surfaces. 
Let $\Gamma=\mathrm{PSL}_{2}(\mathbb{Z})$ denote modular group which we regard as usual as Möbius transformations on the complex plane $\mathbb{C}$ or upper half plane $\mathcal{U}:=\{z \in \mathbb{C}: \operatorname{Im}(\mathrm{z})>0\}$. Let $\Gamma^{*}$ be the group generated by $\Gamma$ and the antiholomorphic reflection $R: z \mapsto-\bar{z}$. We call $\Gamma^{*}$ the extended modular group. It is easy to see that $\Gamma$ is of index 2 in $\Gamma^{*}$.

(3.1) Lemma. The $J$-function $J: \mathcal{U} \rightarrow \mathbb{C}$ induces an isomorphism of Klein surfaces

$$
\mathcal{U} / \Gamma^{*} \stackrel{\sim}{\rightarrow} \Delta \backslash\{\infty\}
$$

Proof. It is well-know that $J$ induces an isomorphism $\mathcal{U} / \Gamma \rightarrow \mathbb{C}$. It therefore suffices to show that the following diagram commutes:

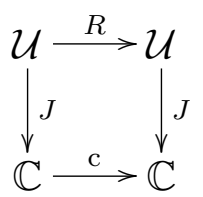

where $c$ denotes complex conjugation. This easily follows from the fact that for every $\tau \in \mathcal{U}$ the lattice $\mathbb{Z}+\mathbb{Z}(-\bar{\tau})$ is equal to the complex conjugate of the lattice $\mathbb{Z}+\mathbb{Z} \tau$.

(3.2) Theorem. A compact Klein surface $S$ admits a Belyi map $\beta: S \rightarrow \Delta$ if and only if $S$ is isomorphic to the compactification $\overline{\mathcal{U} / L}$ of the quotient surface $\mathcal{U} / L$ for some subgroup $L$ of $\Gamma^{*}$ of finite index.

Proof. Let $L$ be a subgroup of $\Gamma^{*}$ of finite index. If $L \subseteq \Gamma$, there is a classical procedure that turns the orbit space $\mathcal{U} / L$ into a Riemann surface and compactifies it. It is well-known that the canonical projection $\overline{\mathcal{U} / L} \rightarrow \overline{\mathcal{U} / \Gamma} \cong \hat{\mathbb{C}}$ is a Belyi map on the Riemann surface $S:=\overline{\mathcal{U} / L}$. Now Lemma (2.4) implies that $S$ also admits a Belyi map when viewed as a Klein surface. If $L \nsubseteq \subseteq \Gamma$ then $L^{+}:=L \cap \Gamma$ is a subgroup of index 2 in $L$. If we choose any element $\sigma$ in $L \backslash L^{+}$then $\sigma$ induces an antiholomorphic involution $\tau$ on $\mathcal{U} / L^{+}$which can be extended to the compactification $\overline{\mathcal{U} / L^{+}}$(denoted $\tau$ again) and the following diagram commutes by Lemma (3.1):

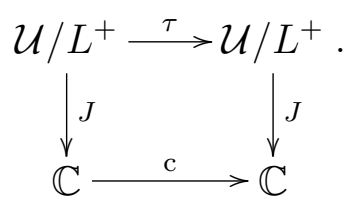

Hence $\overline{\mathcal{U} / L}:=\overline{\mathcal{U} / L^{+}} /\langle\tau\rangle$ is a Klein surface and the $J$-function induces a map $\beta$ : $\overline{\mathcal{U} / L} \rightarrow \Delta$ which is a Belyi map because its complex double $J: \overline{\mathcal{U} / L^{+}} \rightarrow \hat{\mathbb{C}}$ is a Belyi 
map on the Riemann surface $\overline{\mathcal{U} / L^{+}}$.

To prove the converse, let now $\beta: S \rightarrow \Delta$ be a Belyi map. If $S$ is the Klein surface associated with a Riemann surface then $S$ also admits a Belyi map when viewed as a Riemann surface (see Lemma (2.4)), and, by Corollary 2 in [JS2], $S$ is isomorphic to the compactification $\overline{\mathcal{U} / L}$ for some subgroup $L$ of finite index in $\Gamma$, in fact. Otherwise the complex double $S^{\mathrm{c}}$ is connected and $\beta^{\mathrm{c}}: S^{\mathrm{c}} \rightarrow \Delta^{\mathrm{c}}=\hat{\mathbb{C}}$ is a Belyi map. Again by Corollary 2 in [JS2], the Riemann surface $S^{\mathrm{c}}$ is isomorphic to the compactification $\overline{\mathcal{U} / H}$ of $\mathcal{U} / H$ for some subgroup $H$ of $\Gamma$ of finite index. The complex double $S^{\mathrm{c}}$ comes with an antiholomorphic involution $\tau$ such that the following diagram commutes:

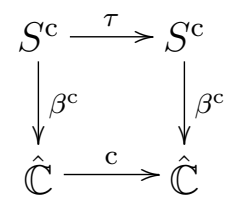

It induces an antiholomorphic involution on $\overline{\mathcal{U} / H}$ (denoted $\tau$ again) which restricts to an involution of the open part $(\mathcal{U} / H)^{o}$ corresponding to $\left(\beta^{\mathrm{c}}\right)^{-1}(\hat{\mathbb{C}} \backslash\{0,1, \infty\})$. Because $H$ acts without fixed points on the preimage $\mathcal{U}^{\circ}$ of $(\mathcal{U} / H)^{\circ}$ the map $\tau$ : $(\mathcal{U} / H)^{o} \rightarrow(\mathcal{U} / H)^{\circ}$ can be lifted to a continuous map $\sigma: \mathcal{U} \rightarrow \mathcal{U}$, see Part $\mathrm{C}$ of Chapter 6 in [Ber]. The map $\sigma$ is antiholomorphic because the canonical projection $\mathcal{U}^{o} \rightarrow(\mathcal{U} / H)^{o}$ is locally biholomorphic. Both $\sigma$ and the reflection $R$ induce the complex conjugation $c$ on $\hat{\mathbb{C}} \backslash\{0,1, \infty\}$ (by Lemma (3.1)). Hence the composition $\sigma \circ R$ is a transformation of the unramified covering $J: \mathcal{U}^{\circ} \rightarrow \hat{\mathbb{C}} \backslash\{0,1, \infty\}$ and therefore an element of the covering group $\Gamma$. Thus the group $L$ generated by $H$ and $\sigma$ is a subgroup of finite index in the extended modular group $\Gamma^{*}$ and we have $S \cong S^{\mathrm{c}} /\langle\tau\rangle \cong \overline{\mathcal{U} / H} /\langle\tau\rangle \cong \overline{\mathcal{U} / L}$ as was to be shown.

For each positive integer $n$ let $\Gamma^{*}(n)$ denote the subgroup of $\Gamma^{*}$ generated by the principal congruence subgroup $\Gamma(n)$ and the reflection $R$. If is easy to see that $\Gamma(n)$ is of index 2 in $\Gamma^{*}(n)$. A fundamental region for the action of $\Gamma^{*}(2)$ on the upper halfplane $\mathcal{U}$ is given by the ideal hyperbolic triangle $\mathcal{T}$ with vertices 0,1 and $\infty$ bounded by the imaginary axis, the line $\operatorname{Re}(z)=1$ and the part of the circle $\left|z-\frac{1}{2}\right|=\frac{1}{2}$ in the upper halfplane. The group $\Gamma^{*}(2)$ is generated by the reflections $c_{0}, c_{1}$ and $c_{\infty}$ in the sides of the triangle $\mathcal{T}$, and the presentation of $\Gamma^{*}(2)$ is

$$
\left\langle c_{0}, c_{1}, c_{\infty} \mid c_{0}^{2}=c_{1}^{2}=c_{\infty}^{2}=1\right\rangle .
$$

Recall the $\lambda$-function $\lambda: \mathcal{U} \rightarrow \hat{\mathbb{C}} \backslash\{0,1, \infty\}$ is defined by

$$
\lambda(\tau)=\frac{\mathfrak{p}_{\tau}\left(\frac{1+\tau}{2}\right)-\mathfrak{p}_{\tau}\left(\frac{1}{2}\right)}{\mathfrak{p}_{\tau}\left(\frac{1+\tau}{2}\right)-\mathfrak{p}_{\tau}\left(\frac{\tau}{2}\right)}
$$


where $\mathfrak{p}_{\tau}$ denotes the Weierstraß $\mathfrak{p}$-function associated with the lattice $\mathbb{Z}+\mathbb{Z} \tau$. The $\lambda$-function turns $\mathcal{U}$ into the universal covering space of $\hat{\mathbb{C}} \backslash\{0,1, \infty\}$ with covering group $\Gamma(2)=\left\langle S_{0}, S_{1}, S_{\infty} \mid S_{0} S_{1} S_{\infty}=1\right\rangle$.

(3.3) Lemma. The $\lambda$-function induces an isomorphism of Klein surfaces

$$
\mathcal{U} / \Gamma^{*}(2) \stackrel{\sim}{\rightarrow} \Delta \backslash\{0,1, \infty\} .
$$

Proof. As in Lemma (3.1) it suffices to check that the diagram

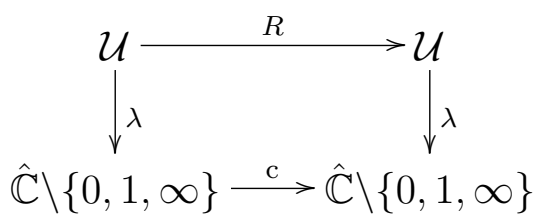

commutes; this is obvious since $\mathfrak{p}_{-\bar{\tau}}(\bar{z})=\overline{\mathfrak{p}_{\tau}(z)}$ and since $\mathfrak{p}_{\tau}$ is double periodic.

(3.4) Theorem. A compact Klein surface $S$ admits a Belyi map $\beta: S \rightarrow \Delta$ if and only if $S$ is isomorphic to the compactification $\overline{\mathcal{U} / M}$ for some subgroup $M$ of $\Gamma^{*}(2)$ of finite index.

Proof. We may consider any subgroup of $\Gamma^{*}(2)$ as a subgroup of $\Gamma^{*}$. Thus one direction is an immediate consequence of Theorem (3.2). The other direction can be proved in the same way as in Theorem (3.2) by replacing the $J$-function by the $\lambda$-function and Corollary 2 in [JS2] by Corollary 1 in [JS2]. (Actually both directions can be proved this way.)

Let $l_{0}, l_{1}$ and $l_{\infty}$ be positive integers or $\infty$. We call the group

$$
\Gamma^{*}\left(l_{0}, l_{1}, l_{\infty}\right)=\left\langle r_{0}, r_{1}, r_{\infty} \mid r_{0}^{2}=r_{1}^{2}=r_{\infty}^{2}=\left(r_{0} r_{1}\right)^{l_{\infty}}=\left(r_{\infty} r_{0}\right)^{l_{1}}=\left(r_{1} r_{\infty}\right)^{l_{0}}=1\right\rangle
$$

an extended triangular group. It acts discontinuously and faithfully on a Riemann surface $\mathcal{X}=\hat{\mathbb{C}}, \mathbb{C}$ or $\mathcal{U}$ as $\sum l_{i}^{-1}>1,=1$ or $<1$. The generators $r_{i}$ correspond to reflections in the sides of a triangle $\mathcal{T}$ with angles $\pi / l_{i}$. We view the triangle group

$$
\Gamma\left(l_{0}, l_{1}, l_{\infty}\right)=\left\langle T_{0}, T_{1}, T_{\infty} \mid T_{i}^{l_{i}}=T_{0} T_{1} T_{\infty}=1\right\rangle
$$

as a subgroup of $\Gamma^{*}\left(l_{0}, l_{1}, l_{\infty}\right)$ via $T_{0}, T_{1}, T_{\infty} \mapsto r_{1} r_{\infty}, r_{\infty} r_{0}, r_{0} r_{1}$. It is easy to see that it is of index 2 with a representative for the nontrivial coset given by any of the reflections $r_{i}$. We recall that there is a holomorphic mapping

$$
j: \mathcal{X} \rightarrow \hat{\mathbb{C}}
$$


mapping the vertices of the triangle $\mathcal{T}$ to 0,1 and $\infty$ and inducing an isomorphism $\mathcal{X} / \Gamma\left(l_{0}, l_{1}, l_{\infty}\right) \stackrel{\sim}{\rightarrow} \hat{\mathbb{C}}$.

(3.5) Lemma. The map $j: \mathcal{X} \rightarrow \hat{\mathbb{C}}$ induces an isomorphism of Klein surfaces

$$
\mathcal{X} / \Gamma^{*}\left(l_{0}, l_{1}, l_{\infty}\right) \stackrel{\sim}{\rightarrow} \Delta .
$$

Proof. As in Lemma (3.1) it suffices to check that the diagram

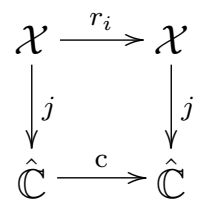

commutes; this immediately follows from the fact that $j$ maps each of the sides of the triangle $\mathcal{T}$ to the real line in $\mathbb{C} \subset \hat{\mathbb{C}}$.

(3.6) Theorem. A compact Klein surface $S$ admits a Belyi map $\beta: S \rightarrow \Delta$ if and only if $S$ is isomorphic to the quotient surface $\mathcal{X} / K$ for some subgroup $K$ of finite index in an extended triangular group.

Proof. Replacing the $J$-function by the $j$-function and the references Lemma (3.1) and Corollary 2 in [JS2] by Lemma (3.5) and Corollary 3 in [JS2], respectively, this can be proved in the same way as in Theorem (3.2).

\section{$\S 4$ Maps on Klein surfaces}

There is a well-developed theory of maps on Riemann surfaces. Without giving precise definitions we remind the reader of the main ideas which are described in detail in [JS1]. Let $S$ be a compact orientable surface. A topological map $\mathcal{M}$ on $S$ is an embedding of a connected graph $\mathcal{G}$ into $S$ such that $S \backslash \mathcal{G}$ is a union of polygonal 2-cells. The graphs may have loops, multiple edges and free edges; these are edges homeomorphic to a closed interval with only one vertex. A dart is an ordered pair consisting of an edge together with an incident vertex. Thus non-free edges contain two darts and the free edges contain one dart, as illustrated in Figure 1, 2 and 3.

Let $\Omega$ denote the set of darts of $\mathcal{M}$. Then there is an involution $x$ defined on $\Omega$ as follows. If $\alpha, \beta$ are darts on a non-free edge, we define $x(\alpha)=\beta$. If $\alpha$ is a dart on a 


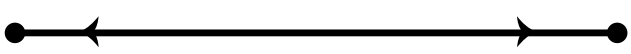

Figure 1:

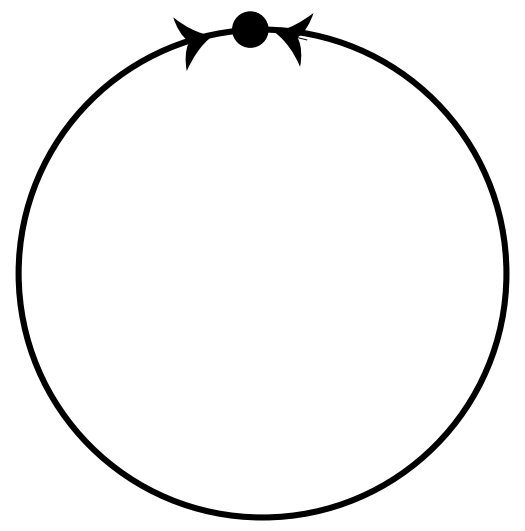

Figure 2:

free edge we define $x(\alpha)=\alpha$. Thus we have a natural 1-1 correspondence between the set of orbits of $\langle x\rangle$ on $\Omega$ and the set of edges of $\mathcal{M}$. Define a permutation $y$ of $\Omega$ that cyclically permutes the darts of $\Omega$ that are incident with a vertex $v$ of $\mathcal{G}$. Precisely, $y$ takes a dart incident with $v$ and transfers it, using the orientation of $S$ to the next dart incident with $v$ in an anticlockwise direction. Thus we have a natural 1-1 correspondence between the set of orbits of $\langle y\rangle$ on $\Omega$ and the set of vertices of $\mathcal{M}$. It is then easy to see that $y^{-1} x$ cyclically permutes the darts around a face in an anticlockwise direction, so that we have the relations $x^{2}=y^{m}=\left(y^{-1} x\right)^{n}=1$, where $m$ is the least common multiple of the vertex valencies and $n$ is the least common multiple of the face sizes. Let $G$ denote the subgroup of the permutation group of $\Omega$ generated by $x$ and $y$. It acts transitively on $\Omega$ as $\mathcal{G}$ is connected.

This motivates us to consider algebraic maps. An algebraic map is a quadruple $(G, \Omega, x, y)$ where $G$ is a group acting transitively and faithfully on a finite set $\Omega$ and $x, y$ are generators of $G$ with $x^{2}=1$. Thus associated with a topological map $\mathcal{M}$ we have an algebraic map $\operatorname{Alg} \mathcal{M}$. In [JS1], we showed how to invert this association and pass from an algebraic to a topological map. We do this by considering the universal topological map $\hat{\mathcal{M}}(m, n)$ of type $(m, n)$. This is the regular map on one of the three

Figure 3: 
simply connected surfaces $\mathcal{X}$ all of whose vertices have valency $m$ and all of whose faces have size $n$. For example, if $m=n=4$, we have the chessboard tessellation of the Euclidean plane. The associated algebraic map $\operatorname{Alg} \hat{\mathcal{M}}(m, n)$ is $(\Gamma, \hat{\Omega}, X, Y)$ where $\Gamma$ is the triangle group

$$
\Gamma(2, m, n)=\left\langle X, Y \mid X^{2}=Y^{m}=\left(Y^{-1} X\right)^{n}=1\right\rangle
$$

acting on $\hat{\Omega}=\Gamma$ via right multiplication. Given an algebraic map $(G, \Omega, x, y)$ of type $(m, n)$ there is an obvious epimorphism $\Gamma \rightarrow G$ and thus $\Gamma$ acts transitively on $\Omega$. Let $M$ be the stabilizer of some element in $\Omega$. Then $M$ is a Fuchsian group and so $\mathcal{X} / M$ is a Riemann surface. Thus with the algebraic map $(G, \Omega, x, y)$ we have associated the Riemann surface $\mathcal{X} / M$. If $(G, \Omega, x, y)$ is the algebraic map associated with a topological map $\mathcal{M}$ on the surface $S$, then $\mathcal{X} / M$ is homeomorphic to $S$; in particular we have turned $S$ into a Riemann surface. We recall that $\mathcal{X} / \Gamma \cong \hat{\mathbb{C}}$ and that the natural projection $\mathcal{X} / M \rightarrow \mathcal{X} / \Gamma \cong \hat{\mathbb{C}}$ is a Belyi function on the Riemann surface $\mathcal{X} / M$.

Vice versa, we can use Belyi functions to construct maps. On $\hat{\mathbb{C}}$ we define the trivial map with one dart with a vertex 0 and free edge along the equator with second endpoint at 1 and a face center at $\infty$. If $\beta: X \rightarrow \hat{\mathbb{C}}$ is a Belyi function on the compact Riemann surface $X$ then we define a map $\mathcal{M}$ on $X$ by using $\beta$ to pull back the trivial map on $\hat{\mathbb{C}}$. Then the Riemann surface $\mathcal{X} / M$ constructed from $\mathcal{M}$ as above is isomorphic to $X$ (see Section 6 in [JS2]).

A theory of maps on (possibly non-orientable) compact surfaces with boundary was developed in [BS]. Also see [JS3]. This was based on the work of [JS1] described above. As above, a topological map $\mathcal{M}$ on a compact surface $S$ with boundary is an embedding of a connected graph $\mathcal{G}$ as above into $S$ with fairly obvious conditions. For instance, an edge of $\mathcal{G}$ not lying on the boundary $\partial S$ can intersect $\partial S$ in at most two points and these cannot be interior points of the edge. At a vertex $p$ on $\partial S$ there may 0,1 or 2 edges emanating from $p$ on $\partial S$. Each face, i.e. connected component of $S \backslash \mathcal{G}$, is homeomorphic to the disc $D=\{z \in \mathbb{C}|| z \mid<1\}$ or to the half disc $E=\{z \in \mathbb{C}|| z \mid<1, \operatorname{Im}(z) \geq 0\}$. First we define a blade to be a half-dart. So an interior dart will have two blades, $b_{1}, b_{2}$, one which points above the edge, and one which points below the edge. If the dart lies on $\partial S$, then the dart will have only one blade. (For a rigorous account see [BS].) We can define three involutions $\tau, \lambda, \rho$ on the set of blades. At a vertex in the interior $S^{o}$, the involution $\tau$ transposes the upper and lower blade and it fixes any blade at a vertex on $\partial S$. For instance $\tau\left(b_{1}\right)=b_{2}$ and $\tau\left(b_{3}\right)=b_{4}$ in Figure 4 .

We call $\tau$ the transverse reflection. The so-called longitudinal reflection $\lambda$ usually 


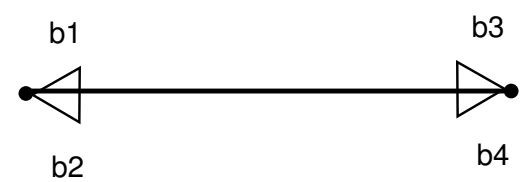

Figure 4:

interchanges the two upper 'upper' blades and also the two 'lower' blades of an edge. For instance $\lambda\left(b_{1}\right)=b_{3}$ and $\lambda\left(b_{2}\right)=b_{4}$ in Figure 4. If the edge intersects the boundary, or if it is a free edge, then $\lambda$ may fix a blade. The last involution we define is the rotary reflection $\rho$. As a blade $b_{1}$ points in a particular direction (either clockwise or anti-clockwise), we follow this direction and if we meet another blade, $b_{5}$, we define $\rho\left(b_{1}\right)=b_{5}$. As blade $b_{5}$ points in the opposite direction, $\rho\left(b_{5}\right)=b_{1}$ as in Figure 6.

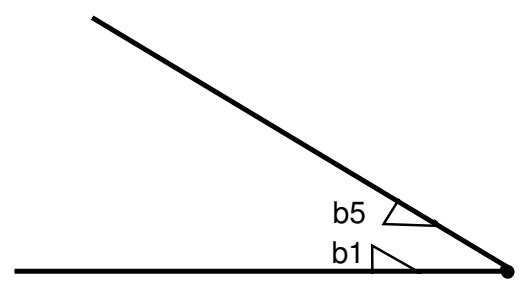

Figure 5:

Again, $\rho$ may fix a blade which corresponds to boundary behaviour.

Notice that $\tau$ and $\rho$ generate a dihedral group that fixes a vertex, $\rho$ and $\lambda$ generate a dihedral group that fixes a face and that $\lambda$ and $\tau$ generate a dihedral group fixing an edge. The group $G^{*}$ generated by $\tau, \lambda$ and $\rho$ obeys the relations

$$
\tau^{2}=\lambda^{2}=\rho^{2}=(\tau \lambda)^{2}=(\tau \rho)^{m}=(\rho \lambda)^{n}=1
$$

where $m$ is the least common multiple of the vertex valencies and $n$ is the least common multiple of the face valencies. In particular there is an obvious epimorphism $\phi: \Gamma^{*}(2, m, n) \rightarrow G^{*}$. Let $K \subseteq \Gamma^{*}(2, m, n)$ denote the stabilizer of a blade. We call $K$ the map subgroup for $\mathcal{M}$. Then $K$ is of finite index in $\Gamma^{*}(2, m, n)$ and the orbit space $\mathcal{X} / K$ is homeomorphic to $S$. By Theorem (3.6), $\mathcal{X} / K$ is a Klein surface and the canonical projection $S=\mathcal{X} / K \rightarrow \mathcal{X} / \Gamma^{*}(2, m, n) \cong \Delta$ is a Belyi function.

To invert this process, we define the trivial map on $\Delta$ : it consists of a single blade; regard $\Delta$ as being a hemisphere with boundary the great circle through $0,1, \infty$; then 
the single blade consists of half a free edge with endpoints a vertex at 0 and a free end point at 1. If $S$ is a Klein surface and $\beta: S \rightarrow \Delta$ is a Belyi map on $S$, then we can pull back the trivial map on $\Delta$ to a map $\mathcal{M}$ on $S$. Let $K$ denote the map subgroup for $\mathcal{M}$. Then we can find an isomorphism between the Klein surfaces $\mathcal{X} / K$ and $S$ such that the following diagram commutes:

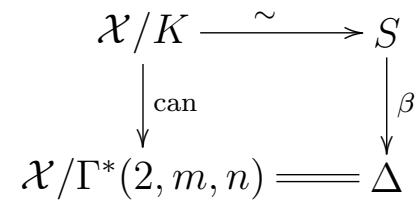

Summarizing we have proved:

(4.1) Theorem. Let $S$ be a compact connected Klein surface. Then the following statements are equivalent:

(a) $S$ admits a Belyi map $\beta: S \rightarrow \Delta$.

(b) $S$ carries a map $\mathcal{M}$ such that $S$ is isomorphic to the quotient surface $\mathcal{X} / K$ where $K$ is the map subgroup for $\mathcal{M}$.

(c) $S$ is isomorphic to the quotient surface $\mathcal{X} / K$ for some subgroup $K$ of finite index in an extended triangle group of the form $\Gamma^{*}(2, m, n)$.

\section{References}

[Al] N. L. Alling, "Real elliptic curves", North-Holland Mathematical Studies 54, North-Holland Publishing Co., Amsterdam (1981).

[AG] N. L. Alling and N. Greenleaf, "Foundations of the theory of Klein surfaces", Lecture Notes in Mathematics 219, Springer-Verlag, Berlin (1971).

[Be] G. V. Belyi, Galois extensions of a maximal cyclotomic field, Izv. Akad. Nauk SSSR Ser. Mat. 43, 267-276 (1979).

[Ber] L. Bers, Quasiconformal mappings and Teichmüller's theorem, in "Analytic functions", Princeton Univ. Press, Princeton, 89-119 (1960).

[BS] R. P. Bryant and D. Singerman, Foundations of the theory of maps on surfaces with boundary, Quart. J. Math. Oxford Ser.(2) 36, 17-41 (1985). 
[Ga] J. M. GamboA, "Compact Klein surfaces with boundary viewed as real compact smooth algebraic curves", Mem. Real Acad. Cienc. Exact. Fs. Natur. Madrid 27, Madrid (1991).

[Go] G. GonzÁlez-Diez, Variations on Belyi’s theorem, Q. J. Math. 57, 339354 (2006).

[EGAIV] A. GrothendiEck, "Éléments de géométrie algébrique. IV. Étude locale des schémas et des morphismes de schémas. II", Inst. Hautes Études Sci. Publ. Math. 24, Bure-sur-Yvette (1965).

[Gr] A. Grothendieck, Esquisse d'un programme, in "Geometric Galois actions", Vol. I, London Math. Soc. Lecture Note Ser. 242, Cambrige Univ. Press, Cambridge, 5-48 (1997).

[JS1] G. A. Jones And D. Singerman, Theory of maps on orientable surfaces, Proc. London Math. Soc. 37, 273-308 (1978).

[JS2] G. A. Jones And D. Singerman, Belyi functions, hypermaps and Galois groups, Bull. London Math. Soc. 28, 561-590 (1996).

[JS3] G. A. Jones And D. Singerman, Maps, hypermaps and triangle groups, in "The Grothendieck theory of dessins d'enfants", London Math. Soc. Lecture Note Ser. 200, Cambridge Univ. Press, Cambridge, 115-145 (1994).

[Kö] B. Köck, Belyi's Theorem revisited, Beiträge Algebra Geom. 45, 253-265 (2004).

[LZ] S. K. LAndo And A. K. Zvonkin, "Graphs on surfaces and their applications", Encyclopaedia of Mathematical Sciences 141, Springer-Verlag, Berlin (2004).

[Mo] C. J. Moreno, "Algebraic curves over finite fields", Cambridge Tracts in Mathematics 97, Cambridge Univ. Press, Cambridge (1991).

[Wo] J. Wolfart, The "obvious" part of Belyi's theorem and Riemann surfaces with many automorphisms, in "Geometric Galois actions", Vol. 1, London Math. Soc. Lecture Note Ser., 242, Cambridge Univ. Press, Cambridge, 97-112 (1997).

School of Mathematics, University of Southampton, Highfield, Southampton SO17 1BJ, United Kingdom.

E-mail: B.Koeck@soton.ac.uk, ds@maths.soton.ac.uk 